\title{
Realization of a New Linear System Explaining the Fundamental Laws of Light and Energy in Infinite Spaces.
}

\author{
Mohamed Daris* \\ Department of Physics, University of Sciences, Morocco
}

*Corresponding Author: Mohamed Daris, Department of Physics, University of Sciences, Morocco

\begin{abstract}
In doing this work I try to better understand and understand relativity by giving it another form that better describes the relation between the energy of a particle and the speed of light and I find a new parameter of time that I use it to explain deeper my theorem which is based on this newly exploiting aspect through a development of a special mathematical concept that gave me specific access to develop and bring out the energy theorem. I would also say that the theorem is a new mathematical and physical structure that are both linking and appropriate the speed of light remains the same my concept just explained the phenomena that we can find them in far galaxies or even in our galaxy, as for example the black hole because at the level of these giant physical bodies matter it exists only in the state of emptiness with enormous speed and infinite time. So I try to give a new concept for this coast.
\end{abstract}

Keywords: new relationship of energies, new fundamental physics, speed of light; fundamental relationship; universal infinite energy; new time parameter.

\section{ABREVIATIONS :}

C:speed of light ; E: the energy of light ; E': new speed of light ; TF: final time parameter ;

m: mass of matter .

\section{INTRODUCTION}

In this study, I put relations energy time and energy speed and material very developed to solve the classic theme of the equation of light and to come to make a new model of the equation of light by the hypothesis of creation of a new time parameter and a new concept which is based on this parameter which is a mathematical equation that I put them to get to make the new linear model of the equation of light in connection with the final time parameter.

\section{DISCUSSION:}

Relationship sutured endless new.

The following new Relationship that we have:

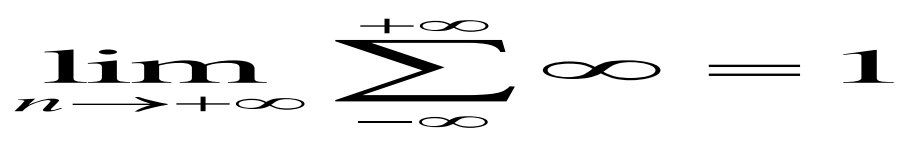

Demonstrations were:

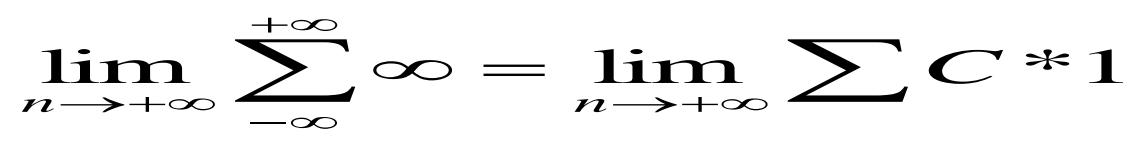

Or:

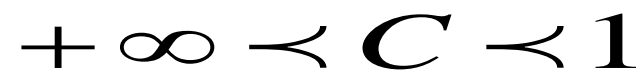

So this implies the following relationship: 


\section{$\lim _{n \rightarrow+\infty} \sum c^{*} 1=1^{+}$}

And:

$$
\lim _{n \rightarrow-\infty} \sum_{-\infty}^{+\infty} \infty=\lim _{n \rightarrow-\infty} \sum c^{* 1} 1
$$

Or we have:

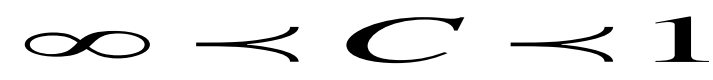

This implies that:

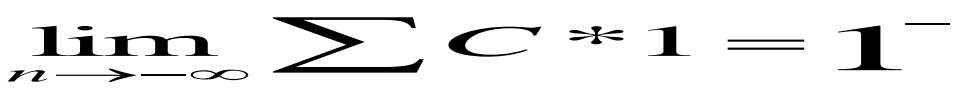

Or we have:

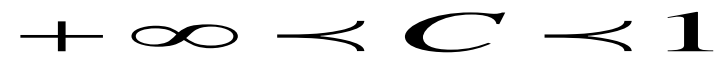

And it was for:

$$
\lim _{n \rightarrow+\infty} \sum_{+\infty}^{-\infty} \infty=\lim _{n \rightarrow+\infty} \sum_{+\infty} C * 1+\lim _{n \rightarrow-\infty} \sum_{-\infty} C * 1+\lim _{n \rightarrow+\infty} \sum_{+\infty} C * 1
$$

So the result is equal to:

$$
\mathbf{1}^{+}+\mathbf{1}^{-}+\mathbf{1}^{+}=\mathbf{O}+\mathbf{1}^{+}=\mathbf{1}^{+}=\mathbf{1}
$$

So we have the end result is:
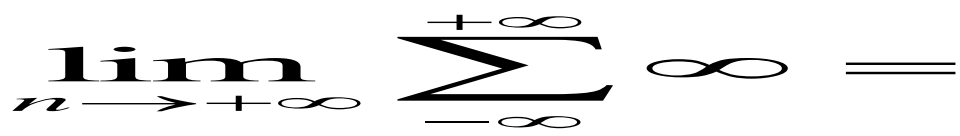

Direct result of the physical fundamental law:

In another demonstration an equation which connect all the universal parameter of the universe between them this equation become:

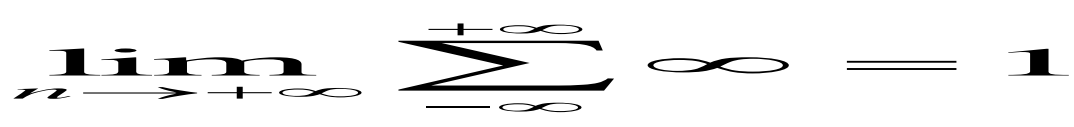

And it is known after the universal relationship:

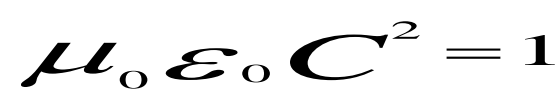

And we:

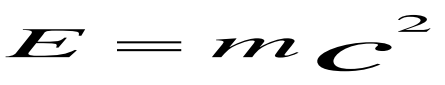

So we:

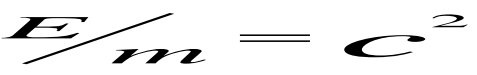

Was:

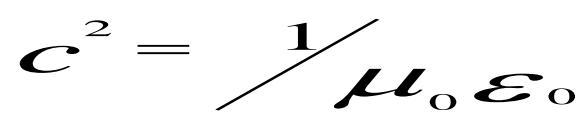


So we:

$$
E / m=1 / \mu_{0} \varepsilon_{0}
$$

So we will:

$$
\mu_{\mathrm{o}} \varepsilon_{\mathrm{o}} E=m
$$

Returning to our equation:

We have:

$$
\begin{aligned}
& \lim \sum \infty=\mu_{0} \varepsilon_{0} C^{2} \\
& \lim \sum \infty=\mu \varepsilon_{0} \varepsilon_{0}^{E} / m
\end{aligned}
$$

And we all at some point the universe tend toward a moment that I called:

$T_{F}$

So we:

$$
\lim \sum \infty=7
$$

So we:

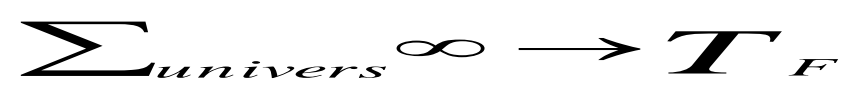

With:

$$
Z_{H}=\mu_{0} E E_{m}
$$

We have:

$$
c^{2}=1 / c_{0} E 0
$$

And:

$$
c^{2}=\frac{\pi}{m}
$$

So we:

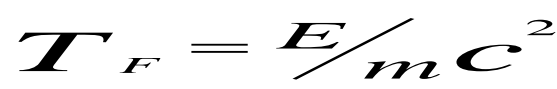

So we:

$$
T+C^{2}=\frac{E}{m}
$$

We set:

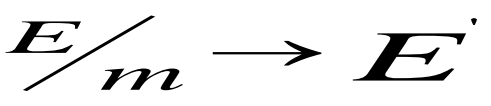

So we:

$$
\boldsymbol{E}^{\prime}=\boldsymbol{T}_{F} \boldsymbol{C}^{2}
$$


And we:

$$
E=m c^{2}
$$

So we will:

$$
c^{2}=\frac{\pi}{2}
$$

And:

$$
C^{2}=E / m
$$

So we:

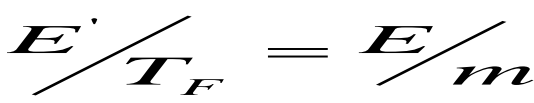

So we have the following general relationship:

$$
E^{\prime} m=T_{F} E
$$

And with:

$$
T_{F}=E / m C^{2}
$$

So we:

$$
E \cdot m=E^{2} / m c^{2}
$$

So:

$$
\Psi^{\prime}=(E / \operatorname{mc})^{2}
$$

And we:

$$
E=T+C^{2}
$$

So:

$$
\prod_{F} C^{2}=(E / m c)^{2}
$$

So:

$$
T_{F} C^{4}=(E / m)^{2}
$$

So we have the following general fundamental relationship:

$$
E=m c^{2} \sqrt{T_{F}}
$$


A relation can be deduced between $\mathrm{E}$ and $\mathrm{E}$ ':

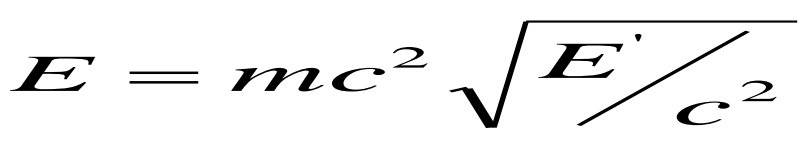

So we:

$$
E=m c \sqrt{E^{\prime}}
$$

Explanation of two theorems (x) and (y) dimension of a physical perspective;

For (x):

I deduced that the time is the universe is relative to the speed and mass of the materiel according to its energy E.

I was always positive because : $\quad \mathbf{m c}^{2} \sqrt{\boldsymbol{T}_{F}} \succ \mathrm{O}$

So we can see that time very high compared to the speed and the materiel itself.

For (y):

We have a new energy that appears after time $T_{F}$ is a universal infinite energy is always positive is proportional to the energy $\mathrm{E}$ and it can reach the whole of the universe and explains the total flow of matter through transformation of these two energy.

Note:

All these calculations are made in order to correct and retrieve a developed and corrective version of the classic Einstein relation and would be linked to anew time parameter that is $T_{F}$.

\section{REFERENCES}

[1] Jonsson B, Nilsson M (2001) Transitive Closures of Regular Relations forVerifying Infnite-State Systems. International Conference on Tools and Algorithms for the Construction and Analysis of Systems.

[2] Araki H (2004) Representations of the Canonical Commutation RelationsDescribing a Nonrelativistic Infnite Free Bose Gas. Journal of Mathematical Physics 4: 637

[3] $\mathrm{Hu}$ J, Wu WZ (2012) Belief and plausibility reducts in incomplete informationsystems with fuzzy decision". Machine Learning and Cybernetics 1: 212-218.

[4] Gilbert Grynberg; Alain Aspect; Claude Fabre (2010). Introduction to Quantum Optics: From the SemiClassical Approach to Quantized Light. Cambridge University Press. p. 341. ISBN 0-521-55112-9. ...deals with the quantum vacuum where, in contrast to the classical vacuum, radiation has properties, in particular, fluctuations, with which one can associate physical effects.

Citation: Mohamed Daris, Realization of a New Linear System Explaining the Fundamental Laws of Light and Energy in Infinite Spaces., International Journal of Scientific and Innovative Mathematical Research (IJSIMR), vol. 8, no. 7, pp. 5-9, 2020. Available : DOI: https://doi.org/10.20431/2347-3142.0807002

Copyright: (C) 2020 Authors. This is an open-access article distributed under the terms of the Creative Commons Attribution License, which permits unrestricted use, distribution, and reproduction in any medium, provided the original author and source are credited. 